\title{
PREPROCESSING ALGORITHM FOR DIGITAL FINGERPRINT IMAGE RECOGNITION
}

\author{
Farah Dhib Tatar ${ }^{1}$ and Mohsen Machhout ${ }^{2}$ \\ ${ }^{1}$ Department of Electrical Engineering \\ National school of the studies of engineer of Tunis, Tunis, Tunisia \\ ${ }^{2}$ Faculty of Sciences of Monastir, Monastir, Tunisia
}

\begin{abstract}
Biometrics is one of the most used technologies in the field of security due to its reliability and performance. It is based on several physical human characteristics but the most used technology is the fingerprint recognition, and since we must carry out an image processing to be able to exploit the data in each fingerprint we propose in this article an image preprocessing procedure in order to improve its quality before extracting the necessary information for the comparison phase.
\end{abstract}

\section{KEYWORDS}

Fingerprint Recognition Algorithm; Image processing; Image sensor; Image filtering

\section{INTRODUCTION}

The reliable identification of people is becoming more and more necessary to solve problems of access and identity theft [1].

Access control is a requirement especially to sensitive places that require a high level of confidentiality. The identification keys are of three main types: keys based on the knowledge of passwords or encrypted codes, others based on the possession of access cards badges, keys, etc., or we can identify us based on the physical characteristics of person: these are biometric identification techniques

Unlike the first two methods that can pose serious falsification problems, biometric identification techniques are directly related to people's physical characters and therefore can not be copied or desired.

The goals of a biometric security system can be multiple. A security system can, for example, use the tools that biometrics makes available to manage the security of logical access of a system. This is probably the most common use of biometrics. But it can also be used to secure the physical access of a room containing sensitive data, from any place on which we choose to install a biometric lock*.

*: http://www.agent-de-securite.be/biometrie/securite_biometrique.html 
The biometric identification techniques are various: we can analyze the shape of the hand [2], the design of the iris [3], the voice, the vascularization of the retina and the shape of the face. [4] Likewise, the dynamic recognition of the signature [5], analyzed in real time (speed, pressure on the pen, etc).

Given its low cost, ease of operation and reliability of fingerprint recognition results, this technology occupies more than two-thirds of the biometric market.

Fingerprint recognition algorithms are diverse and are based on different techniques in order to extract useful information from the input image. Obviously, the clearer and better the image, the more reliable and fast the system is. An image sensor is composed of many photosites, each corresponding to a picture element, it is the "pixel".Each pixel of an image sensor records the amount of light to which it is exposed and converts it into a corresponding number of electrons. The stronger the light, the higher the number of electrons generated.

Sensors embedded in fingerprint recognition systems are diverse and based on several technologies, noting for example:

$>$ CCD sensors: they have been used in cameras for over 30 years and have many advantages. In general, they still offer a slightly higher sensitivity to light than CMOS sensors and produce a little less noise. However, they are more expensive and more difficult to integrate into a camera.

$>$ CMOS sensors: they reduce the overall price of cameras, as they contain all the elements necessary for the composition of cameras. They offer more integration possibilities and more functions than CCD sensors. They also offer shorter measurement times, lower power dissipation at the chip, and reduced footprint.

$>$ Megapixel sensors (sensors containing one million pixels or more): They are integrated with megapixel cameras and are of a size equal to or only slightly larger than VGA sensors that provide a resolution of $640 \times 480(307,200)$ pixels. This means that the size of each pixel on a megapixel sensor is smaller than that on a VGA sensor. Therefore, although the megapixel camera provides higher resolution and more detail, it is less sensitive to light than its VGA counterpart since the pixel size is smaller and the light reflected from an object is spread over more pixels.

Whatever the technology used, the purpose of a fingerprint recognition system is to have reliable authentication and fast error rate as small as possible. For this purpose, we present in this paper a preprocessing procedure of the captured image in order to increase its quality before proceeding to the extraction step of useful information and consequently increase the overall performance of the system by minimizing the total processing time as well as the rate of erroneous results.

\section{PREPROCESSING PROCEDURE FOR DigitAl FingeRPRINT RECOGNITION}

Digital fingerprint recognition is the most widely used biometric technique because of its ease of use and demonstration. The chains of recognition by digital processes are based on image 
processing techniques making it possible to extract the useful information of each image (fingerprint) in order to be able to identify it and consequently compared it to other fingerprints.

Our fingerprints are unique, at least on certain points: these are called minutiae, that is to say, the lines, the bifurcations, the "islets", the points, the ending of the peaks. The design formed by these lines on our fingers never evolves. Depending on the level of security desired, it is estimated that between 10 and 20 points of correspondence are required to certify a fingerprint.

\section{A. Description of the fingerprint}

A fingerprint consists of a set of locally parallel lines forming a single pattern for each individual (Figure 1), there are streaks (or ridges, these are the lines in contact with a surface to the touch) and the grooves (this are the hollows between two streaks). **

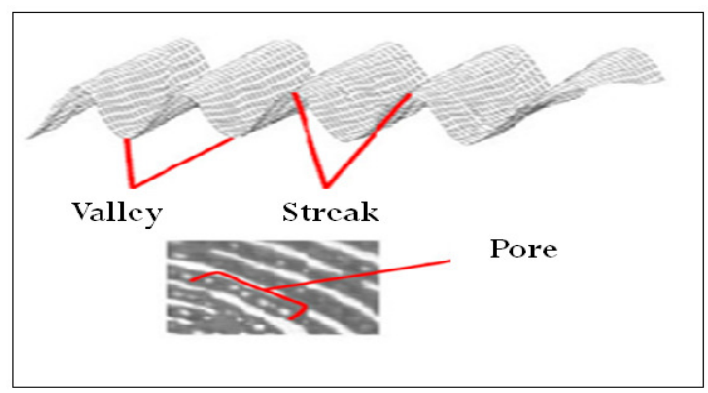

Fig. 1. Fingerprint characteristic

Each imprint has a set of global (centers and deltas) and local (minutiae) singular points (Figure 2).

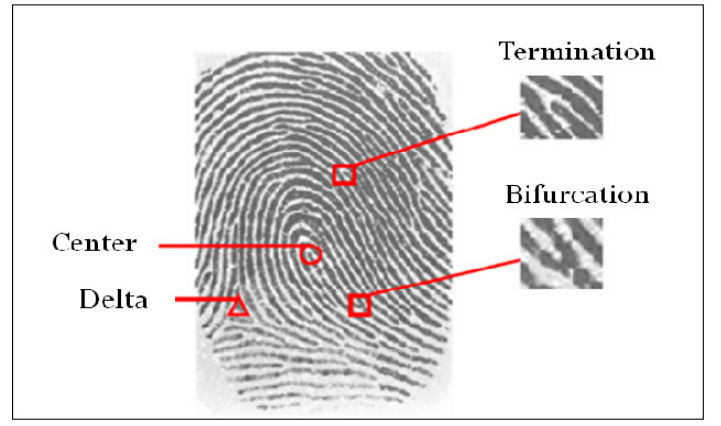

Fig. 2. Local and Global singular points in a fingerprint

The centers correspond to places of convergence of the striations while the deltas correspond to places of divergence.

Fingerprint recognition algorithms use several filtering methods in order to locate the useful points for each fingerprint. The most used filter is that of Gabor given its proven performance in this type of treatment.

\footnotetext{
**http://www.orcanthus.com/main/product/product.php?idpr=4
} 
However, in order to have reliable results, the input image of the filter must be as clear as possible, for this reason we propose a series of mathematical and morphological operations to improve the appearance of the image before pass to the filtering step.

When capturing a fingerprint image, several factors affect image quality such as:

$>$ Scars in the finger, age of the person.

$>$ Parasitic substances (water, grease, dirt, etc.)

$>$ Capture environment: temperature (dilation, compression), humidity

These various factors greatly affect the quality of the input image of the system and consequently increase the error rate and reduce the overall performance of the application; We must therefore perform a specific treatment in order to reduce the parasitic effects and improve the image quality used. The different pretreatment phases are presented in Figure 3.

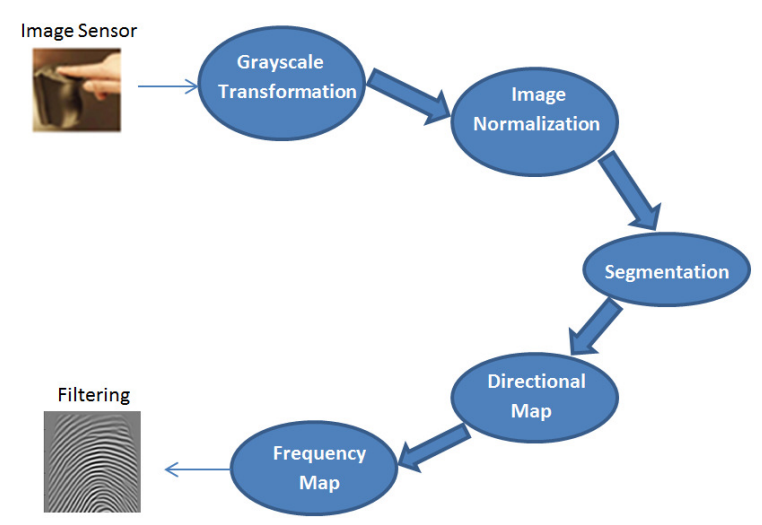

Fig. 3. Preprocessing steps

\section{B. Grayscale transformation}

A fingerprint sensor in general returns a color image. For this algorithm, there is no need for color schemes for processing. We must therefore make a transformation into grayscale. This transformation allows us to reduce the space used for recording data included in the image; in fact, the color pixels require 24 bits to be able to represent the different levels of colors, on the other hand in grayscale we only need 8 bits for each pixel since we have only 256 gray levels. this operation is performed according to the following formula (1):

$$
\mathrm{I}=(\mathrm{R}+\mathrm{G}+\mathrm{B}) / 3
$$

Where I is the value of the pixel in gray and $\mathrm{R}, \mathrm{G}$ and $\mathrm{B}$ are the values of the color components.

\section{Image normalization}

The normalization operation makes it possible to increase the contrasts in the image by making a certain adjustment to the gray levels represented in each pixel without affecting the useful 
information included in the fingerprint image.This operation is done locally on each block according to the following steps:

\section{Calculation of the average:}

$$
M=\frac{1}{n \times m} \sum_{i=0}^{n-1} \sum_{j=0}^{m-1} I(i, j)
$$

I $(i, j)$ is the value of the pixel $(i, j)$ and $M$ is the value of the average of the image while $m$ and $n$ are the dimensions of the image.

$>$ Calculation of variance:

$$
V=\frac{1}{n \times m} \sum_{i=0}^{n-1} \sum_{j=0}^{m-1}(I(i, j)-M)^{2}
$$

$\mathrm{V}$ is the variance of the image.

$>$ Calculation of the standardized gray level value of the pixel $(i, j)[6]$ :

$$
N(i, j)= \begin{cases}M_{0}+\sqrt{\frac{V_{0} \times(I(i, j)-M)^{2}}{V}} \text { If } I(i, j)>M \\ M_{0}-\sqrt{\frac{V_{0} \times(I(i, j)-M)^{2}}{V}} & \text { If } I(i, j)<M\end{cases}
$$

M0 and V0 are the desired values for mean and variance, respectively. We chose $\_0=100$ and $\mathrm{V} 0=100$.

Normalization does not change the structure of the image, but it is used to standardize the variation of gray levels.

\section{Segmentation}

This operation aims to minimize the noise present in the image as well as to center the useful information that will be used in the comparison phase of the different signatures, so we eliminate the noisy areas as well as the useless edges of the image because they can lead us to make false comparisons.

The results of this step is a binary image of the same size as the original image and called "Mask", the block corresponding to the bottom of the image will be filled with 0 in the mask, and the rest will be filled by 1 .This is not all to satisfactorily because many blocks containing no useful information persist.

To avoid this we decided to eliminate the blocks belonging to the bottom of the image according to their average value. The local average value of each block $\mathrm{Ml}$ is calculated and compared to a threshold T:

If $\boldsymbol{M}_{\boldsymbol{l}}<T$ then we consider that the block contains no useful information and it is eliminated. 
If $\boldsymbol{M}_{\boldsymbol{l}}>T$ then the block contains part of the fingerprint and is retained.

The threshold $\mathrm{T}$ has been chosen according to the average value $\mathrm{M}_{\mathrm{g}}$ of the overall image such that $=\frac{\mathrm{M}_{\mathrm{g}}}{2}$.

The mask then undergoes two treatments:

Any non-noisy block with at least 3 adjacent blocks defined as noisy is eliminated (this corresponds to a strongly noisy area or the edge of the fingerprint.

The set of blocks at the edge of the image is deleted.

An example of segmentation is shown in Figure 4 [7].

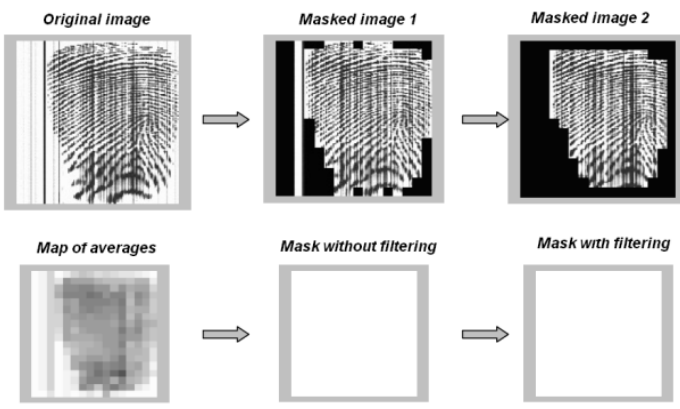

Fig. 4. Effect of segmentation on the image

\section{E. Spatial estimate of the directional map}

To obtain the directional map, we goes through two stages: the estimation of the orientation and the smoothing of the directional map.

\section{1) Estimation of the orientation:}

The directional map present the orientation of each streak contained in the fingerprint.Orientation estimation is a fundamental step in Gabor's image-based image enhancement process. (Figure 5).

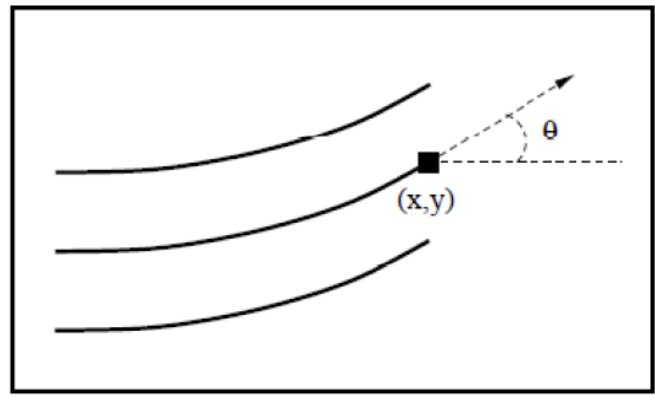

Fig. 5. Local orientation of a pixel 
We present here the optimized calculation of the directional map of the fingerprint [7]. This measurement must be reliable because, in the case of directional filtering, a poor evaluation of the direction would cause a degradation of the image. We use here the method based on the least square approximation ([8], [9]) which is very effective in the case of very noisy images.

To calculate the direction associated with a pixel $\mathrm{x}, \mathrm{y}$ of the image, we consider its neighborhood $\mathrm{V}$ of size W x W pixels. We calculate the gradients, $\boldsymbol{G}_{\boldsymbol{x}}(\boldsymbol{i}, \boldsymbol{j})$ in x and $\boldsymbol{G}_{\boldsymbol{y}}(\boldsymbol{i}, \boldsymbol{j})$ in y for each pixel i, $\mathrm{j}$ of neighborhood V. For the calculation of the gradient, the use of Sobel masks proved to be the most efficient in the case of our images. Thus the local direction in neighborhood $\mathrm{V}$ is estimated by the following relation (the proof of this formula is detailed in the article of M. Bazen and $\mathrm{H}$. Gerez [10].

$$
\begin{gathered}
\theta(x, y)=\frac{1}{2} \tan ^{-1}\left(\frac{\sum_{i=1}^{w} \sum_{j=1}^{w} 2 . G_{x}(i, j) \cdot G_{y}(i, j)}{\sum_{i=1}^{w} \sum_{j=1}^{w}\left(G_{x}^{2}(i, j)-G_{y}^{2}(i, j)\right)}\right) \\
\text { with } G_{x}=V(x, y) \times\left[\begin{array}{ccc}
-1 & 0 & 1 \\
-2 & 0 & 2 \\
-1 & 0 & 1
\end{array}\right] \\
\text { and } G_{y}=V(x, y) \times\left[\begin{array}{ccc}
-1 & -2 & -1 \\
0 & 0 & 0 \\
1 & 2 & 1
\end{array}\right]
\end{gathered}
$$

It is thus possible to calculate the local direction at each pixel of the image but this is very expensive in computation time, so it is much more efficient to divide the image into adjacent blocks and evaluate the local mean direction to the block leaves to interpolate the direction for each pixel.

\section{2) Refinement of the directional map}

In the case where the input image is too noisy we can have false results when detecting the local directions of the pixels. Thus we can note the presence of vertical lines at the directional map levels that have no direction.

An example of the presence of vertical lines is illustrated in the figure (6) presenting the case of estimation of the directional map with an "Arch" type fingerprint.

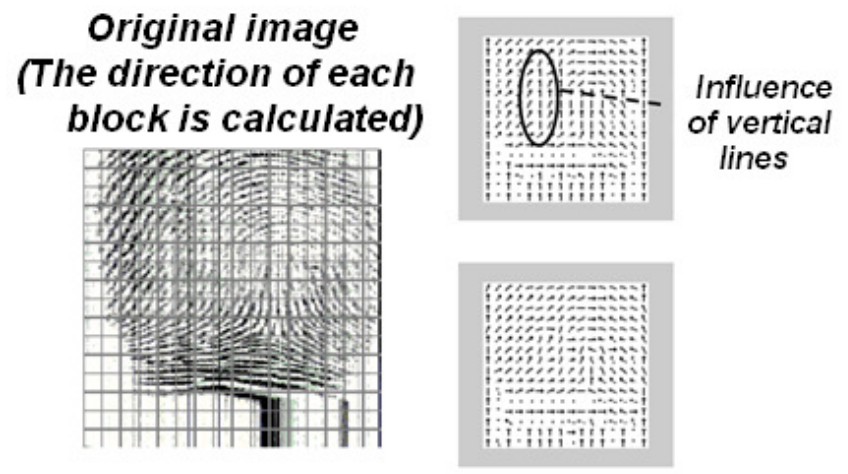

Fig. 6. Influence of vertical lines 
This then causes a very large angular variation between two adjacent blocks. However, a fingerprint has some directional continuity, such a variation between two adjacent blocks is then representative of a poor estimate. To eliminate such discontinuities, a low-pass filter is applied to the directional map. Applying a low-pass filter requires that the orientation of the image be converted into a continuous vector field. This vector field has as components $\mathrm{x}$ and y respectively defined by:

$$
\varphi_{x}(i, j)=\cos (2 . \theta(i, j)) \text { and } \quad \varphi_{y}(i, j)=\sin (2 . \theta(i, j))
$$

With the two components of the vector obtained, one can apply the Gaussian low pass filter of size W_ $\theta \times \mathrm{W}_{-} \theta$ defined by:

$$
\begin{gathered}
\varphi_{x}^{\prime}(i, j)=\sum_{u=\frac{-w_{\theta}}{2}}^{\frac{w_{\theta}}{2}} \sum_{v=\frac{-w_{\theta}}{2}}^{\frac{w_{\theta}}{2}} G(u, v) \cdot \varphi_{x}(i-v \cdot w, j-v \cdot w) \\
\varphi_{y}^{\prime}(i, j)=\sum_{u=\frac{-w_{\theta}}{2}}^{\frac{w_{\theta}}{2}} \sum_{v=\frac{w_{\theta}}{2}}^{\frac{w_{\theta}}{2}} G(u, v) \cdot \varphi_{y}(i-v \cdot w, j-v \cdot w)
\end{gathered}
$$

Where $\mathrm{G}$ is the Gaussian low pass filter and $\mathrm{w}$ is the block size.

Finally, the local orientation smoothed at the pixel $(i, j)$ is given by:

$$
O(i, j)=\frac{1}{2} \tan ^{-1} \frac{\varphi_{y}^{\prime}(i, j)}{\varphi_{x}^{\prime}(i, j)}
$$

The Figure (7) shows the example of the estimation of the directional map for different types of fingerprint.

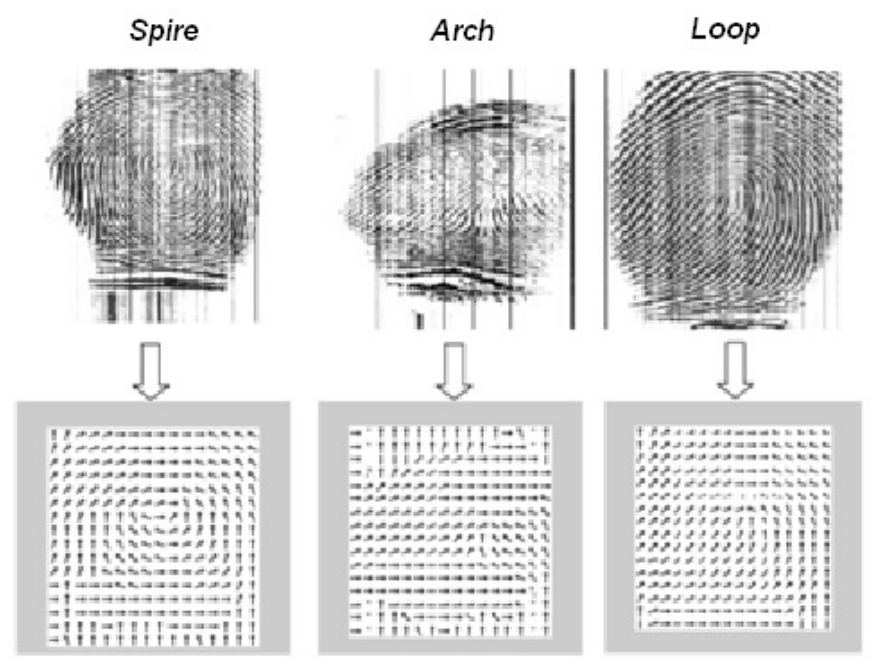

Fig. 7. Estimation of the directional map for different types of fingerprint 
The frequency map of the image consists in estimating the local frequency of the striations in each pixel.

\section{1) Frequencyof each block}

The frequency mapis an image of the same size as that of the fingerprint and represents the local frequency of the streaks. The first thing to do is to divide the image into sub-blocks of size $\mathrm{W} \times \mathrm{W}$. Then, we have to project each pixel in the block orthogonal to its direction (that is to say in the direction $\left(\frac{\pi}{2}-\theta\right)$ ), we obtain a vector V presenting a set of extrema (figure 8) [10].

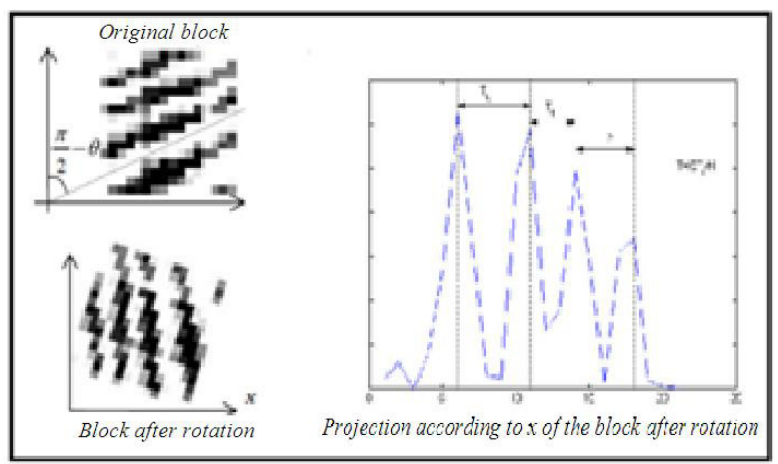

Fig. 8. Extrema obtained after projection

\section{2) Spatial estimation of frequency}

In the extrema vector obtained, the maxima represent the centers of the striations and the minima correspond to the centers of the valleys. The inter-striated local period is then estimated by calculating the average distance between two consecutive maxima $S(i, j)$ :

$$
F(i, j)=\frac{1}{S(i, j)}
$$

The maxima $M \_i$ and the minima $m_{-} i$ are determined by detecting the zero crossing of the derivative of the vector $\mathrm{V}$, which makes it possible to obtain the sequence $\left\{M \_1, m \_1, \ldots . . . ., M \_k, m \_k, M \_(k+1)\right\}$. If the difference between a maximum $M \_i$ and a minimum $\mathrm{m} \_\mathrm{i}$ is less than a threshold $\mathrm{T}$ then we consider that $\mathrm{M} \_\mathrm{i}$ corresponds to a noise and it is eliminated.

If the resultant vector contains at least two maxima then the inter-strikethrough period is calculated by averaging the distances between two consecutive maxima, otherwise the period takes the value of zero. When the estimated period is zero this means that the block contains no streaks (background image) or that it is too noisy to reliably estimate the local frequency.

\section{RESULTS AND DISCUSSION}

We used Matlab to validate all this steps; the results are shown in the following pictures: 
International Journal of Computational Science and Information Technology (IJCSITY) Vol.6, No.1/2/3, August 2018 A. Applying the mask on the image:
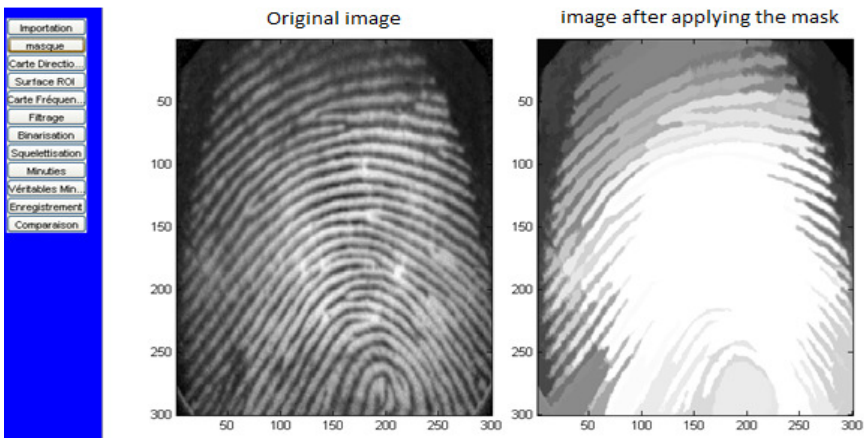

Fig. 9. Mask of the image

This step allows us to eliminate the edges of the image as well as the very noisy areas.

\section{B. Extraction of the directional map}
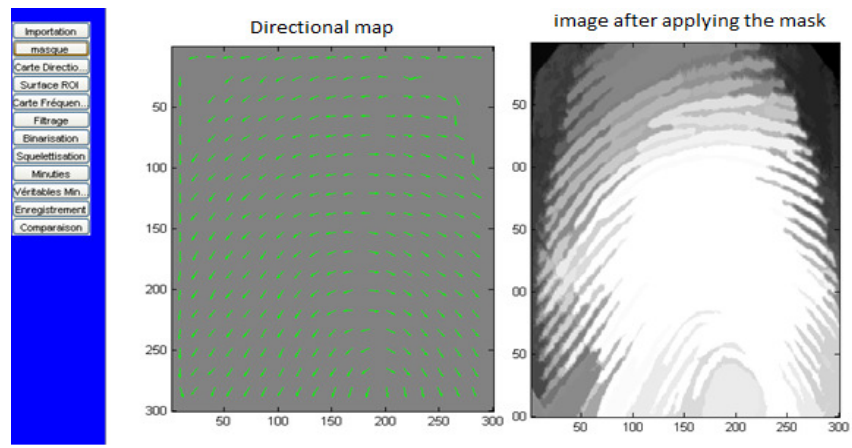

Fig. 10. Directional map of the fingerprint

The directional map defines the local orientation of streaks contained in the fingerprint. C. Extraction of the frequency card
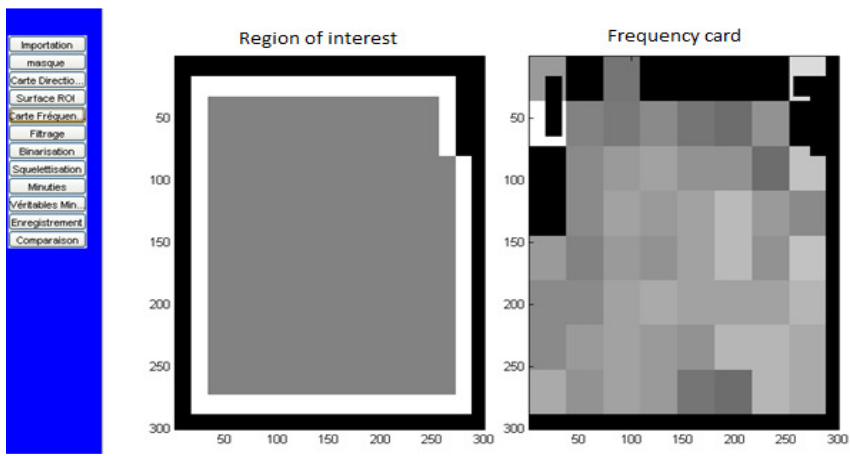

Fig. 11. Frequency map of the fingerprint 
The frequency map estimates the local frequency of the streaks in each pixel.

In order to highlight the importance of image preprocessing we have considered a database based on a hundred images captured by different types of sensors, in the first place we tried to go directly to the stage filtering without making any changes to the images, we extracted the useful information from each fingerprint, and then we passed to the authentication phase.

Second, we went through the preprocessing of images before filtering and the steps that follow; the different results are displayed in the following table:

Table 1. Result of Preprocessing Application

\begin{tabular}{|c|c|c|}
\hline & $\begin{array}{c}\text { Image without } \\
\text { pretreatment }\end{array}$ & $\begin{array}{c}\text { Images with } \\
\text { preprocessing }\end{array}$ \\
\hline $\begin{array}{c}\text { Total number of } \\
\text { images }\end{array}$ & 100 & 100 \\
\hline $\begin{array}{c}\text { Rate of } \\
\text { erroneous } \\
\text { results }\end{array}$ & 46 & 22 \\
\hline
\end{tabular}

We therefore note that thanks to the various operations carried out to improve the quality of the images, the rate of the erroneous results is reduced by $24 \%$.

The improvements noted make the system more efficient from a point of view of reliability; the criterion of speed can not be discussed in this step since the total processing time depends directly on the type of processor used and therefore it varies according to the hardware platform used to implement the fingerprint recognition algorithm.

\section{CONCLUSION}

Some are the type of sensor used or the algorithm principle applied; the image quality to be processed remains a very important factor that directly affects the overall system performance.

For this fact, we have proposed in this article a set of operations to apply to the image in order to increase its quality to be able to facilitate the different phases of treatment that follow.

The results obtained are motivating in terms of total error rate reduction, however, the total processing time remains directly related to the type of hardware platform used.

\section{REFERENCES}

[1] Debnath Bhattacharyya, Rahul Ranjan1, Farkhod Alisherov A., and Minkyu Choi, Biometric Authentication: A Review, International Journal of u- and e- Service, Science and Technology, Vol. 2, No. 3, September, 2009.

[2] Aythami Morales, Miguel A. Ferrer, Carlos M. Travieso and Jesus B. Alonso "Multisampling approach applied to contactless hand biometrics" International Carnahan Conference on Security Technology (ICCST), IEEE 2012. 
International Journal of Computational Science and Information Technology (IJCSITY) Vol.6, No.1/2/3, August 2018

[3] Aly I. Desoky, Hesham A. Ali and Nahla B. Abdel-Hamid "Enhancing iris recognition system performance" The International Conference on Computer Engineering \& Systems. 2010.

[4] Navaneeth Bodla, Jingxiao Zheng, Hongyu Xu, Jun-Cheng Chen, Carlos Castillo and Rama Chellappa "Deep Heterogeneous Feature Fusion for Template-Based Face Recognition" IEEE Winter Conference on Applications of Computer Vision (WACV). 2017.

[5] Gang Li, Rui Zhang, Matthew Ritchie and Hugh Griffiths "Sparsity-based dynamic hand gesture recognition using micro-Doppler signatures" IEEE Radar Conference (RadarConf).2017.

[6] R. Stefanelli and A. Rosenfeld, Some parallel thinning algorithms for digital pictures , Journal of the ACM, Vol.18, No2, pp.255-264, April 1971.

[7] P.B. Wamelen, Z. Li and S.S. Iyengar, "A fast expected time algorithm for the 2-D point pattern matching problem", Pattern Recognition, Vol. 37, No. 8, pp. 1699-1711, August 2004.

[8] N. GALY « Etude d'un système de reconnaissance d'empreintes digitales pour un capteur microsystème à balayage» : http://tima.imag.fr/publications/files/th/2006/sff_207.pdf, 2005.

[9] G.O. Williams, Iris Recognition Technology , IEEE Aerospace and Electronics Systems Magazine, Volume 12, Issue 4, pp. 23 -29, April 1997.

[10] A.M. Bazen and S.H. Gerez, "Directional Field Computation for Fingerprints Based on the Principal Component Analysis of Local Gradients", Veldhoven, Netherland, November 2000 